\title{
On the Hipparcos photometry of chemically peculiar B, A, and F stars $^{\star}$
}

\author{
Saul J. Adelman \\ Department of Physics, The Citadel, 171 Moultrie Street, Charleston SC 29409, U.S.A. \\ e-mail: adelmans@citadel.edu
}

Received February 23; accepted March 20, 1998

\begin{abstract}
The Hipparcos photometry of the Chemically Peculiar main sequence B, A, and F stars is examined for variability. Some non-magnetic CP stars, MercuryManganese and metallic-line stars, which according to canonical wisdom should not be variable, may be variable and are identified for further study. Some potentially important magnetic CP stars are noted.
\end{abstract}

Key words: stars: early type — stars: chemically peculiar

\section{Introduction}

Photometry from the Hipparcos Satellite (European Space Agency (ESA) 1997) can be used to study stellar variability. This absolute photometry database contains about 100 observations for each measured star with most of the observations bunched into several intervals. I investigate the Chemically Peculiar stars of the Upper Main Sequence except for the $\lambda$ Boo stars. Single normal B, A, and F main sequence band stars for the most part are non-variable. Exceptions include the $\beta$ Cephei, 53 Persei, and slowly pulsating $\mathrm{B}$ stars in the $\mathrm{B}$ stars and the $\delta$ Scuti stars in the late $\mathrm{A}$ and early $\mathrm{F}$ stars.

Whether or not the non-magnetic CP stars: the Mercury-Manganese (HgMn) stars and the metallic-line $(\mathrm{Am})$ stars (which are not also $\delta$ Scuti stars) are intrinsically constant still remains to be determined as the literature contains claims of low amplitude variability for some of these stars, many of which have been refuted for the better known stars. Still a few claims for variability might be valid. In looking for stars which might be investigated further to settle this question, I used both the

Send offprint requests to: S.J. Adelman

* Tables 1,2, and 3 are available only in electronic form at the CDS via anonymous ftp to cdsarc.u-strasbg.fr (130.79.128.5) or via http://cdsweb.u-strasbg.fr/Abstract.html standard error as given in the Hipparcos Catalogue and the "amplitude", the difference between the 95 th and 5 th percentile magnitudes from its Photometry Annex. The Hipparcos Catalogue gives the scatter (one-half the difference between the 85 th and 15 th percentile magnitudes). Although the scatter and amplitude should correlate with one another and with the standard error, the amplitude might be more sensitive to potentially eclipsing systems. Tables 1, 2, and 3 give the Hipparcos photometry for the $\mathrm{HgMn}, \mathrm{Am}$, and magnetic CP stars, respectively. For each star, two identifiers are normally given, the HR or HD number and the Hipparcos Catalogue number, the mean Hipparcos magnitude, its standard error, its amplitude, and variability comments including the period if known except for the magnetic CP stars.

The standard error, the amplitude, and the scatter tend to increase with mean magnitude. These values are due to the intrinsic variability of the star as well as to errors in particular those due to photon statistics. Hence a noise model is needed in investigating variability. When one plots the standard error vs. the mean Hipparcos magnitude of the HgMn stars (Fig. 1), there is a lower bound at each magnitude which is consistent with that for the two other types of stars which are studied in this paper. The smallest standard errors occur among the HgMn stars at $0.0003 \mathrm{mag}$. If one looks instead at normal stars, then the smallest standard errors are often 0.0002 mag (Adelman 1998). The values of the lower bound increase with magnitude as would be expected due to photon statistics with the value for each magnitude increasing by 1.58 and the value at 5 th magnitude being 0.0002 mag. These values are given in Table 4.

Usually for each type of star, the standard errors are proportional to the amplitude as seen in Fig. 2 for the $\mathrm{HgMn}$ stars. But there are some stars which appreciably deviate from the mean relation such as the eclipsing $\mathrm{HgMn}$ star binary AR Aurigae. As some CP stars are more variable in particular wavelength ranges than others, using 


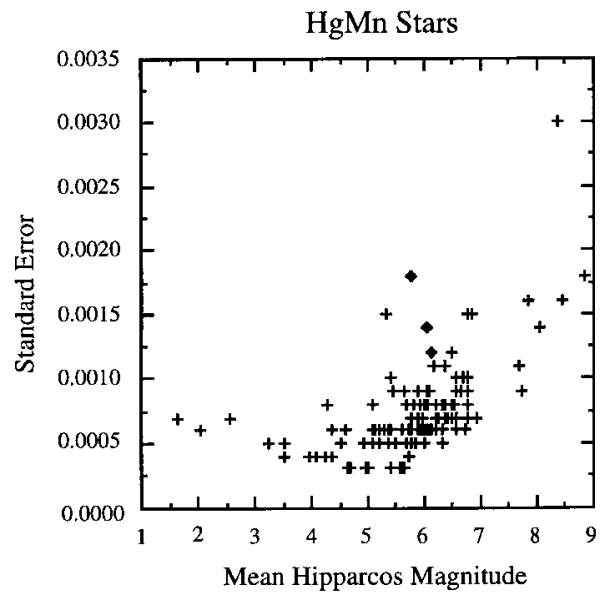

Fig. 1. The standard error vs. mean Hipparcos magnitude diagram for the HgMn stars. Closed diamonds indicate three definitely variable stars according to Hipparcos photometry. The other stars are indicated by plus signs

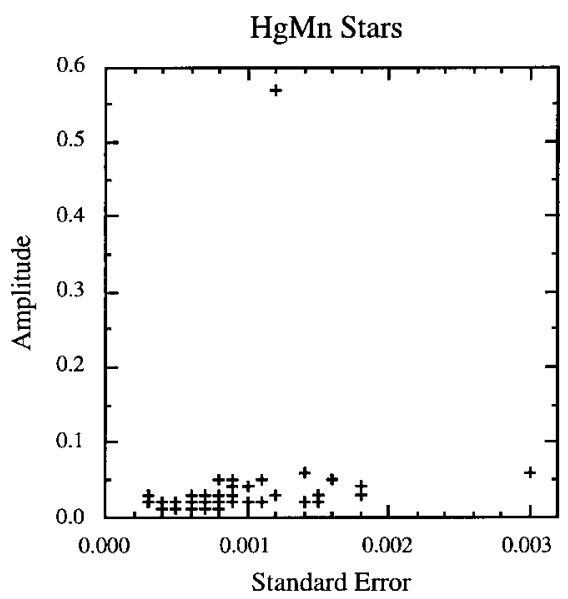

Fig. 2. The amplitude vs. standard error diagram for $\mathrm{HgMn}$ stars

Table 4. Noise model lower bounds to the standard error

\begin{tabular}{cc}
\hline $\begin{array}{c}\text { mean magnitude } \\
\text { (magnitude) }\end{array}$ & $\begin{array}{c}\text { Lower Bound } \\
\text { (magnitude) }\end{array}$ \\
\hline less than 5 & 0.00020 \\
6 & 0.00032 \\
7 & 0.00050 \\
8 & 0.00079 \\
9 & 0.00125 \\
10 & 0.00197 \\
\hline
\end{tabular}

Hipparcos magnitudes to search for photometric variability may not find all such stars.

\section{Mercury-Manganese stars}

Adelman (1993) and Adelman et al. (1994) showed that several Mercury-Manganese (HgMn) stars, including the most often cited as variable, were non-variable both by examining published and Four College Automated Photoelectric Telescope filter photometry. A list of HgMn stars (Table 1) was compiled mainly from Aikman (1976), Wolff \& Preston (1978), the 5 th edition of the Yale Bright Star Catalog (Hoffleit \& Warren 1991), and Renson (1991). Some stars which were not true class members were removed by consulting the literature especially Abt \& Morrell (1995) and Garrison \& Gray (1994). To classify a star as a HgMn star usually requires a photographic region spectrogram with a resolution greater than that typically used for classification purposes and often with a resolution similar to that needed for an abundance analysis. The paucity of known HgMn stars fainter than about 7 th magnitude is due to this cause. There is also the unresolved problem of whether the HgMn stars have weak magnetic fields.

Figure 1 shows the relation between standard error and mean magnitude for the HgMn stars. Most HgMn stars are constant according to the Hipparcos Catalogue. Only four were found to have periods. Three are in the region defined by the variable normal stars: AR Aur (= HR 1728), a well known eclipsing binary, HR 7911, and 13 And (= HR 8913). The Hipparcos photometry light curves for AR Aur and 13 And well fit their respective photometry, but that for HR 7911 could be improved. According to Hoffleit (1982), HR 7911 is a close binary star and 13 And is a spectroscopic binary. HR 6023 (= $\phi$ Her) with a small amplitude is noted as variable. Its range of magnitudes is 4.219 to $4.226 \mathrm{mag}$ and its standard error is $0.0004 \mathrm{mag}$. These values strongly suggest that this star is constant. Thus both HR 7911 and 13 And deserve additional study.

If we use as a guide that other possible HgMn star variables should have distances from the constancy line at least as great as the three variable $\mathrm{HgMn}$ stars found by the Hipparcos team, then additional candidates are: HR 149, HR 8512, HD 2019, HD 65949, and HD 65950.

Catalano \& Leone (1991) found HR 149 (= HD 3322) to be a variable star with a period of 4.6904 days while Aikman (1976) discovered it to be a spectroscopic binary with a period of about 400 days. On the other hand Winzer (1974) noted it was constant to better than 0.01 mag during a 14 day observing run. Its Hipparcos photometry indicates a scatter with an amplitude of about 0.03 mag.

Stickland \& Weatherby (1984) derived a binary orbit for HR 8512. Abt \& Morgan (1969) classified both HD 65949 and HD 65950 as HgMn stars. Abt \& Levy (1972) found the former was a SB1 star while North (1984) used the later as a comparison star for differential photometry.

Figure 2 shows the relation between standard error and amplitude for the HgMn stars. The discrepant position of AR Aurigae was previously noted. 


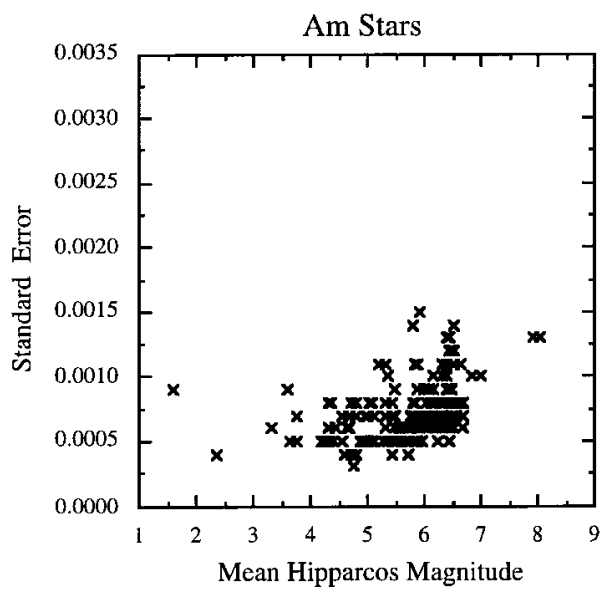

Fig. 3. The standard error vs. mean Hipparcos magnitude diagram for the Am stars

\section{The Am stars}

The sample of Am stars is from Abt \& Morrell (1995) supplemented by stars that I found were hot Am stars by performing abundance analyses using spectrograms taken at the Dominion Astrophysical Observatory (see Adelman 1994; Caliskan \& Adelman 1997 and references therein). Am stars which were $\delta$ Scuti stars or which were in systems with variable companions were excluded. In the sample which remained, there were some variable stars. Most are eclipsing binaries: GW Eri (= HD 26591), RR Lyn (= HD 44691), WW Aur (= HD 46052), S Ant (= HD 46810), CO Cam (= HD 106112), V624 Her (= HD 86809), KO Aql (= HD 92117), DV Aql (= HD 199603), and AN And (= HD 219815) while IW Per (= HD 21912) is an ellipsoidal variable. These stars were moved to the bottom of Table 2 and their values were not plotted in the diagrams. Figure 3 shows the standard error vs. mean magnitude plot of the remaining stars and Fig. 4 the standard error vs. amplitude relation. In the former plot HD 201671 was excluded and HD 133408 in the later. The later star's position in the last plot may be due to one discrepant value.

Comparing Figs. 1 and 3, which were plotted with similarly scaled axes, shows generally similar distributions and lower bounds at each magnitude. Four stars are possible variables HD 27628 (=60 Tau), HD 30453 (= HR 1528), HD 124915 (= HR 5341), and HD 223991 (= HR 9044). For the Am stars, as there are known eclipsing binaries, we must keep this possibility in mind. Corbally (1987) believes contrary to Abt \& Morrell (1995) that HR 9044 is a composite between A5 IVn and A2 Vn stars which on the aggregate looks like an Am star. The only remaining star with a period in Table 2 HD 16769 is a spectroscopic binary.

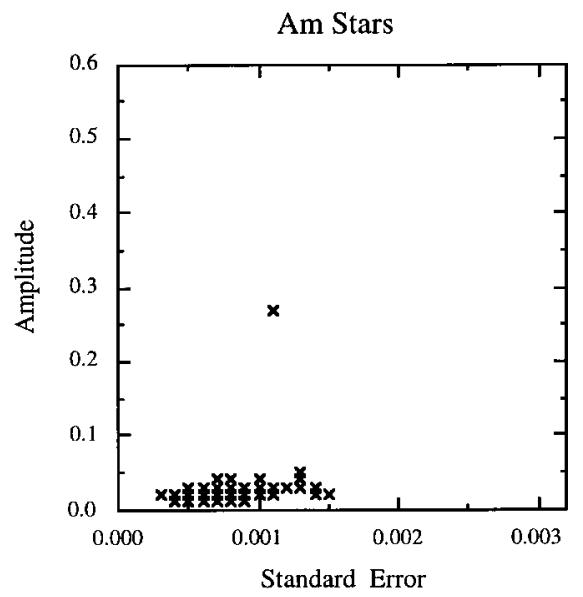

Fig. 4. The amplitude vs. standard error diagram for Am stars

\section{The magnetic CP stars}

The list of magnetic CP (MCP) stars comes from the period compilation of Catalano \& Renson (1997 and earlier references therein) supplemented by other stars of interest. Figure 5 shows the standard error vs. mean magnitude plot with the left panel showing the full distribution and the right panel showing the plot with the same ranges as Figs. 1 and 3. The MCP stars are simply more variable than the non-magnetic CP stars. But their standard error vs. amplitude plot (Fig. 6) is the same in the part in common.

To be able to detect the variability of such stars by differential photometry, one needs to use comparison and check stars which are more constant. Adelman (1998) showed that finding appropriate stars with amplitudes of $0.02 \mathrm{mag}$ and standard errors of $0.0008 \mathrm{mag}$ or less was usually possible. Thus most MCP stars should be found to be variable. Some apparently constant MCP stars will be found to be variable when observed over a time period greater than the approximately three years of Hipparcos observations. The Hipparcos data confirm that some MCP stars such as HD 204411 (= HR 8216) have been essentially constant stars during the working lifetime of the Hipparcos satellite.

Table 3 should be very useful for observers of MCP stars who want to be sure ahead of time that their observing programs will yield good results. Many of the brighter stars with amplitudes greater than $0.02 \mathrm{mag}$ already have good periods, but this is not generally true of fainter stars with such variability. At a given apparent magnitude it is somewhat easier to define the shapes of the light curves as the amplitude of variability increases. Stars with large amplitude variability probably have relatively large magnetic fields. If their periods are sufficiently short (of order a day), they should be candidates to look for precessional effects (Shore \& Adelman 1976). 
Magnetic Chemically Peculiar Stars

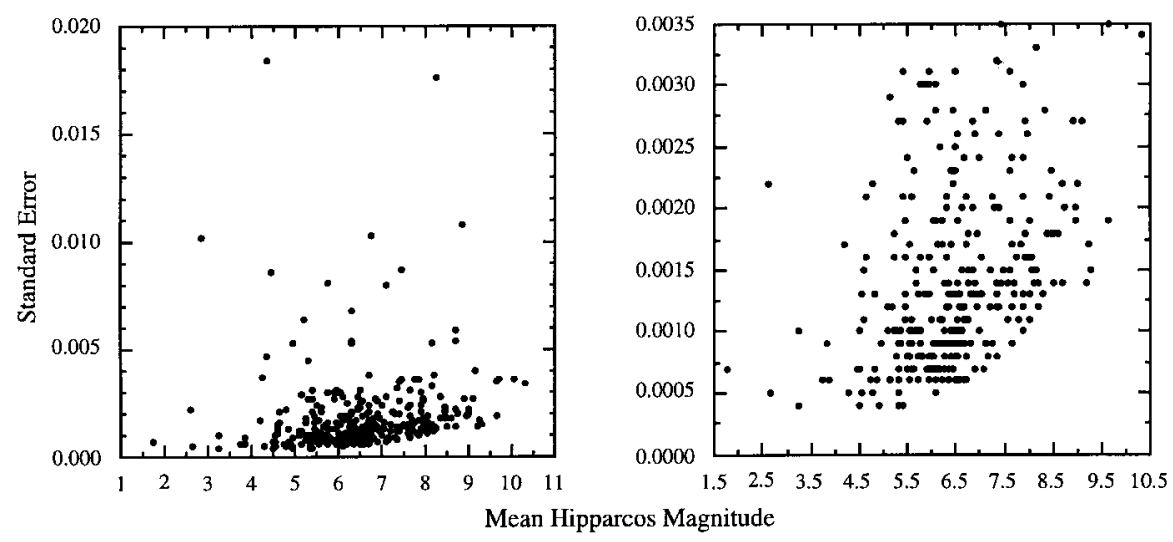

Fig. 5. The standard error vs. mean Hipparcos magnitude diagram for the MCP stars. The left panel shows the full range of values while the right panel is sized comparable to Figs. 1 and 3. Comparison of these diagrams shows that one can construct a common lower bound as a function of magnitude for all three kinds of star

Magnetic Chemically Peculiar Stars
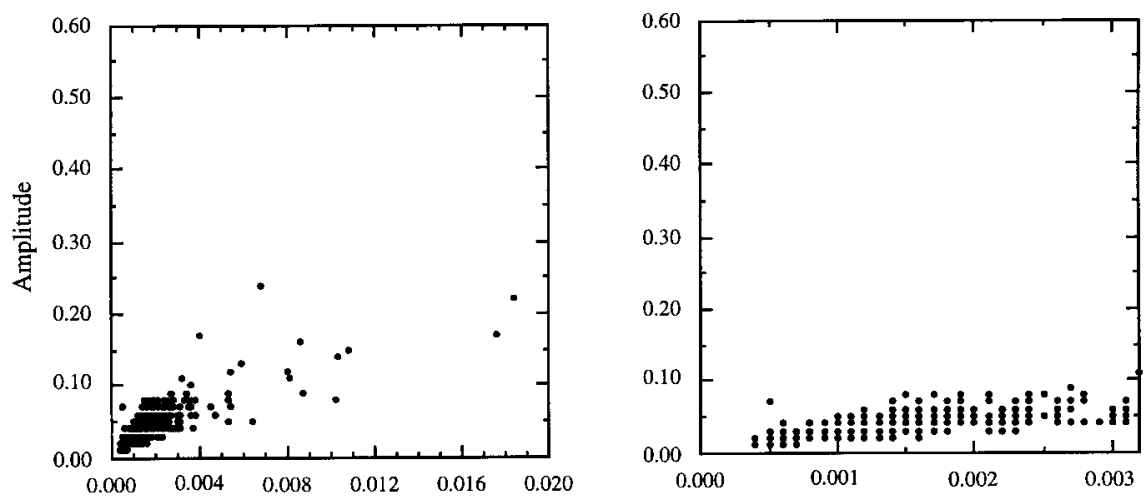

Standard Error

Fig. 6. The amplitude vs. standard error diagram for MCP stars. The left panel shows the full range of values while the right panel is sized comparable to Figs. 2 and 4. Comparison of these diagrams shows that the same correlation probably applies to $\mathrm{HgMn}, \mathrm{Am}$, and MCP stars

\section{Comments}

The Hipparcos photometry indicates that the Am stars as a class are a slightly better candidate for internal constancy than the HgMn stars. To resolve the constancy questions requires careful study of the indicated stars as well as those whose positions in the standard error vs. mean magnitude diagrams are close to them. It is critical to be certain that any photometric variability is not due to eclipses or to variable companions. If for either class, the indicated stars or others are found to be intrinsically variable and true class members, then the question is resolved. If they can be excluded, then stars which are less variable via Hipparcos photometry will have to be studied until one reaches the point where constancy can be assured by comparison with other classes of stars whose members are intrinsically constant.
Acknowledgements. I thank Dr. Robert J. Dukes Jr., for some useful discussions of stellar variability and Dr. Diane Pyper Smith for recommending some MCP stars to be examined.

\section{References}

Abt H.A., Levy S.G., 1972, ApJ 172, 355

Abt H.A., Morrell N.I., 1995, ApJS 99, 135

Abt H.A., Morgan W.W., 1969, AJ 74, 813

Adelman S.J., 1993, A\&A 259, 411

Adelman S.J., 1994, MNRAS 266, 97

Adelman S.J., 1998, Baltic Astron. 7 (in press)

Adelman S.J., Brown B.H., Caliskan H., Reese D.F., Adelman C.J., 1994, A\&AS 106, 333

Aikman G.C.L., 1996, Pub. Dom. Ap. Obs. 14, 379

Caliskan H., Adelman S.J., 1997, MNRAS 288, 501

Catalano F.A., Leone F., 1991, A\&A 244, 327

Catalano F.A., Renson P., 1997, A\&AS 121, 57

Corbally C.J., 1987, ApJS 63, 365 
European Space Agency, 1997, The Hipparcos and Tycho Catalogues, SP- 1200

Hoffleit D., 1982, The Bright Star Catalogue, 4 th revised edition, Yale University Observatory, New Haven, CT

Hoffleit D., Warren W.H. Jr., The Bright Star Catalogue, 5 th revised edition, ADC Selected Astronomical Catalogs, Vol. 1, NASA

Garrison R.F., Gray R.O., 1994, AJ 107, 1556
North P., 1984, A\&AS 55, 259

Renson P., 1991, Catalogue des Étoiles Ap \& Am, Institut d'astrophysique, Universite de Liège, Liège

Shore S.N., Adelman S.J., 1976 ApJ 209, 816

Stickland D.J., Weatherby J., 1984, A\&AS 57, 55

Winzer J.E., 1974, PhD Thesis, University of Toronto

Wolff S.C., Preston G.W., 1998, ApJS 37, 371 